\title{
Bertolotti Syndrome: A Diagnostic and Management Dilemma for Pain Physicians
}

\author{
Departments of Anesthesia, *Nuclear Medicine, \\ Sanjay Gandhi Postgraduate Institute of Medical Sciences, Lucknow, India
}

Anuj Jain, MD, Anil Agarwal, MD, Suruchi Jain, MD*, and Chetna Shamshery, MD

\section{Background:}

Bertolotti's syndrome (BS), a form of lumbago in lumbosacral transitional vertebrae, is an important cause of low back pain in young patients. The purpose of this study was to assess the etiology of low back pain and the efficacy of treatment offered to patients with BS.

\section{Methods:}

All patients of BS Castellvi type1a during a period of 6 months were enrolled in the study. The patients underwent interventional pain procedures for diagnosis and pain relief. Response to the therapy was assessed based on VAS and ODI scores. A 50\% decrease in VAS score or a VAS score less than 3 would be considered adequate pain relief.

\section{Results:}

All 20 patients diagnosed with BS during the 6-month observation period had scoliosis. Common causes of back pain were the ipsilateral L5-S1 facet joint, neoarticulation, the SI joint, and disc degeneration. Responses to various interventions for pain relief were different and inconsistent from patient to patient. In particular, responses to interventions for neoarticular pain were generally poor.

\section{Conclusions:}

Pain in patients with BS does not usually respond to interventional pain treatment. A very dynamic treatment approach must be pursued while managing BS patients, and the treatment plan must be individualized at various stages in order to obtain satisfactory pain relief. (Korean J Pain 2013; 26: 368-373)

Key Words:

Bertolotti's syndrome, low back pain, scoliosis.

Received May 21, 2013. Revised July 10, 2013. Accepted July 12, 2013

Correspondence to: Anuj Jain, MD

Department of Anesthesia, Sanjay Gandhi Postgraduate Institute of Medical Sciences, Lucknow 226014, India

Tel: +91-9415456980, Fax: +91-9415456980,E-mail: anuj.jain.mln@gmail.com

(ㄷ) This is an open-access article distributed under the terms of the Creative Commons Attribution Non-Commercial License (http:// creativecommons.org/licenses/by-nc/3.0/), which permits unrestricted non-commercial use, distribution, and reproduction in any medium, provided the original work is properly cited.

Copyright (C) The Korean Pain Society, 2013 


\section{INTRODUCTION}

The term lumbosacral transitional vertebra (Bertolotti's syndrome) refers to a total or partial unilateral or bilateral fusion of the transverse process of the lowest lumbar vertebra to the sacrum. Bertolotti's syndrome (BS) is an important cause of low back pain (LBP) in young patients. The syndrome affects $4 \%$ to $8 \%$ of the population [1]. BS is characterized by anomalous enlargement of the transverse processes of the most caudal lumbar vertebra, which may articulate or fuse with the sacrum or ilium and cause isolated L4-5 disc disease.

The causes of back pain in BS are multifactorial. Most of the affected patients have scoliosis. The abnormal mechanical stress leads to facet joint arthropathy, as well as iliopsoas and quadratus lumborum strain. Nerve root compression due to narrowing of the intervertebral foramina by the enlarged fan-shaped transverse foramina may lead to neurogenic claudication. An increased prevalence of disc protrusion or extrusion in the disc above the transitional L5 vertebra has been found in patients with LBP. A decreased prevalence of disc protrusion or extrusion was found in the disc below the transitional vertebra [1].

Diagnosis of BS is based on radiological findings and their correlation with the clinical presentation. Plain X-rays of the lumbosacral spine in anteroposterior view are usually sufficient. Radicular features may necessitate an MRI for evaluation of prolapsed intervertebral disc (PIVD), which may $\mathrm{co}^{-}$occur.

The purpose of this study was to assess the etiology of LBP in BS and the efficacy of treatment offered to patients with BS.

\section{MATERIALS AND METHODS}

The present study was performed after obtaining approval from the Institutional Review Board. Patients diagnosed with BS based on radiological findings ( $\mathrm{X}$-ray of the lumbosacral spine, bone scan, MRI, or CT scan) were selected for the study.

As per our departmental protocol, all patients complaining of low backache with a clinical diagnosis of sacroiliitis or facet joint arthropathy underwent bone scan. SPECT/CT was also performed if the initial bone scan revealed any abnormal findings. All patients diagnosed with BS during a period of 6 months were enrolled in the study.
The patients underwent interventional pain procedures according to the clinical diagnosis of the symptoms.

A backache that was located in the midline with aggravation on forward bending and axial loading was labeled as discogenic pain. LBP situated slightly paramedian and increasing on back extension and lateral rotation was labeled to be of facet joint origin. LBP with a positive Patrick's test, Gaenslen's test, and Yeoman's test was labeled as sacroiliitis. Neuralgic pain following a dermatomal pattern of leg pain was labeled as PIVD.

Diagnostic facet joint injection was performed intraarticularly under fluoroscopic guidance and differential diagnostic blocks were given using $1 \mathrm{ml}$ of lignocaine $2 \%$ and bupivacaine $0.5 \%$. A decrease in pain intensity by more than $80 \%$ was considered to be a successful diagnostic block. After identification of the painful facet joint, RF ablation of the medial branch (MB) of the same level and one level superior was performed using a $10 \mathrm{~cm}$ RF needle with a $10 \mathrm{~mm}$ active tip. Proper placement of the needle was confirmed using sensory-motor stimulation testing. Sensory stimulation positive at less than $0.5 \mathrm{mV}$ was considered adequate, while contraction of the multifidus muscle at less than $2.0 \mathrm{mV}$ was considered confirmatory. Once the needle placement was confirmed, continuous $\mathrm{RF}$ ablation was performed for 90 seconds at $90^{\circ} \mathrm{C}$.

SI joint injection was performed under fluoroscopy, with needle placement done in the lower $2 \mathrm{~cm}$ of the SI joint. Differential diagnostic blocks were performed using $1 \mathrm{ml}$ of lignocaine $2 \%$ and bupivacaine $0.5 \%$. A decrease in pain intensity by more than $80 \%$ was considered a successful diagnostic block, after which depomedrol $40 \mathrm{mg}$ was given intra-articularly. Following a successful diagnostic block, RF denervation of the painful SI joint was performed. RF ablation was done using a $10 \mathrm{~cm}$ RF needle with a $10 \mathrm{~mm}$ active tip. Continuous RF was performed at $90^{\circ} \mathrm{C}$ for 90 seconds.

Transformational (TF) steroid injections were given using a subpedicular approach. Injections contained depomedrol $40 \mathrm{mg}$ in $0.25 \%$ bupivacaine, and an interval of at least 4 weeks was maintained between consecutive injections. A maximum of 3 injections were given to obtain the maximum therapeutic benefit of $\mathrm{TF}$ steroid injections.

Provocative discography was done for confirmation of discogenic pain. After needle placement in the intervertebral disc, provocation of discogenic pain was performed by injecting nonionic contrast diluted in normal saline. The 
pressure of the injection was guided by a manometer. A severe concordant pain (VAS greater than 7) at less than 15 psi above opening pressure (AOP) was considered confirmatory, while concordant pain at 15-50 psi AOP was considered equivocal. The adjacent intervertebral discs were also tested for discogenic pain.

Rami communicantes $(\mathrm{RC})$ block was done for palliation of discogenic pain. RC block was placed to target the RC nerves at the vertebral levels above and below the target intervertebral disc. Needle placement was according to the technique described by Tae et al. [2] and diagnostic injection was given using $0.25 \%$ bupivacaine. For L5-S1 discogenic pain, and in cases where more than one intervertebral disc was found to be painful, $\mathrm{RC}$ block was performed at the L1 and L2 vertebral levels [3]. At least 50\% pain relief was considered an adequate response to $\mathrm{RC}$ block. Following an adequate response to $\mathrm{RC}$ block, radiofrequency ablation of the $\mathrm{RC}$ was done using a $15 \mathrm{~cm} \mathrm{RF}$ needle with a $10 \mathrm{~mm}$ active tip. After sensory stimulation, continuous thermal RF was performed at $90^{\circ} \mathrm{C}$ for 90 seconds.

Nucleoplasty was offered to patients with lumbar PIVD with a contained intervertebral disc. This procedure was performed using ArthroCare's SpineWand (ArthroCare ${ }^{\circledR}$ SpineWand $^{\circledR}$, ArthroCare Corp., Austin, TX, USA). After proper cannulae placement, six channels were made by rotating the nucleoplasty wand and using a 10-second coblation cycle.

In patients who had disruption of the annulus fibrosus in discography characterized by spillage of the radiocontrast into the epidural space, nucleoplasty was deferred. Patients who had neurogenic claudication or neuralgic pain in the lower limb as the only symptom of PIVD underwent diagnostic dorsal root ganglion (DRG) block using bupivacaine $0.25 \%$. After confirming the appropriate level, pulsed $\mathrm{RF}$ (pRF) was performed at $42^{\circ} \mathrm{C}$ for 4 minutes using a 10 $\mathrm{cm}$ RF needle with a $10 \mathrm{~mm}$ active tip.

In cases in which multiple etiologies of LBP were inferred, the procedures were planned based on the algorithmic approach for clinical management of chronic spinal pain [4].

Patients who had undergone any previous surgery for LBP, as well as pregnant females, were excluded from the study. Patients lost to follow-up were considered as dropouts. The patients underwent interventional pain procedures according to the clinical diagnosis of the symptoms (Table 1).

Etoricoxib 120 mg tablets once daily were used to provide relief for nociceptive pain. Pregabalin $75 \mathrm{mg}$ twice daily and amitriptyline $10 \mathrm{mg}$ at bedtime were prescribed for patients whose pain had a neuropathic component. Patient follow-up was done at 1, 2, and 4 weeks and thereafter at monthly intervals until 6 months after the procedure.

On the initial visit to the pain clinic and at the subsequent follow-ups, patient pain was assessed for up to 6 months using the visual analog scale (VAS) score, and the physical disability was assessed with the help of Oswestry Disability Index (ODI). Follow-up data was gathered at the personal visit to the OPD or by telephone interview.

\section{RESULTS}

20 patients with $\mathrm{BS}$ were identified out of a total of 365 patients with LBP attending the pain clinic. Out of these 20 patients, 14 were diagnosed based on X-ray, while the remaining 6 were diagnosed following SPECT/CT. In 4 patients, adequate pain relief could not be achieved even after placement of multiple diagnostic blocks. Two patients were lost to follow-up, while 2 patients were managed medically. The median age of the affected pa-

Table 1. Interventions Performed Based on the Most Probable Clinical Diagnosis

\section{Clinical diagnosis}

Facet joint pain

Neo-articulation pain

Discogenic pain

SI joint pain

Neurogenic claudication and

lumbar radicular syndrome
Intervention performed

Diagnostic facet followed by medial branch (MB) radiofrequency (RF) ablation

Diagnostic neo-articular injection followed by steroid injection

Provocative discography, diagnostic rami communicantes (RC) block followed by RF ablation of RC Diagnostic SI injection, RF denervation if positive

Transforaminal (TF) steroid injection, DRG pRF for uncontained intervertebral disc 
tients was 45.6 years. Scoliosis was the most consistent feature, which was observed in all the patients. The next most common feature was ipsilateral L5-S1 joint pain.

In most cases, the clinical diagnosis was confirmed by placement of diagnostic blocks (Table 2). Two patients initially suspected to have L5-S1 facet joint pain experienced relief after placement of ipsilateral SI joint block (Table 2). All patients with confirmed facet joint arthropathy $(n=10)$ had satisfactory relief for up to 1 month following MB RF. In 3 of those 10 patients, the procedure had to be repeated at 3 months, after which the pain relief was restored up until the end of the follow-up period (Table 3). Among the patients undergoing steroid injection in the neoarticulation, one patient experienced pain relief lasting up to 1 month while the other experienced pain relief lasting 3 months. None of the patients, however, experienced pain relief lasting to the end of the 6-month study period. Two of the four patients with neoarticulation pain did not receive any benefit from the treatment being offered and were eventually lost to follow-up (Table 3 ).

All 4 patients undergoing SI joint RF denervation experienced pain relief lasting 6 months (Table 3). Two pa- tients undergoing $\mathrm{RC} \mathrm{RF}$ had good pain relief lasting 6 months. The 2 patients in whom the discogenic pain could not be confirmed with diagnostic RC were managed medically for pain palliation. Both of the patients undergoing nucleoplasty experienced good pain relief even at 3 and 6 months. Two patients undergoing DRG pRF had satisfactory relief for up to 3 months, while only 1 patient experienced relief persisting to the end of 6 months (Table 3 ). During the follow-up period, there was a decrease in the disability scoring and VAS scoring of the patient from a more severe group to a less severe group, which persisted until the end of the 6 months (Fig. 1, 2).

\section{DISCUSSION}

Bertolotti's syndrome (BS) is not an uncommon diagnosis in patients with refractory low back pain. The incidence in the present study was found to be $5.4 \%$. The data presented in this study intends to highlight certain important facts regarding the management of BS.

The first challenge is to identify the patient with Bertolotti's syndrome. Plain X-ray of the lumbosacral

Table 2. Clinical Diagnosis and It's Confirmation With Diagnostic Blocks

\begin{tabular}{lccc} 
& & \multicolumn{2}{c}{ Diagnostic blocks } \\
& Clinical diagnosis & Confirm & Failed \\
\cline { 4 - 4 } & & 8 & 2 \\
Ipsilateral facet joint pain & 10 & 2 & 0 \\
Contralateral L5S1 facet pain & 2 & $4^{*}$ & 0 \\
Sacroilitis & 2 & 2 & 2 \\
Discogenic pain & 4 & 2 & 2 \\
Neoarticulation pain & 4 & 2
\end{tabular}

*2 patients clinically diagnosed as ipsilateral facet were relieved of pain after SI joint injection.

Table 3. Tabulated Data Showing the Effects of Interventions on Pain Relief

\begin{tabular}{|c|c|c|c|c|}
\hline \multirow{2}{*}{ Procedures } & \multirow{2}{*}{ Number of patients } & \multicolumn{3}{|c|}{ Duration of pain relief } \\
\hline & & 1 month & 3 month & 6 month \\
\hline MB RF & 10 & 10 & 7 & $10^{*}$ \\
\hline Neoarticulation pain & $4^{\ddagger}$ & 1 & 1 & 0 \\
\hline SI RF denervation & 4 & 4 & 4 & 4 \\
\hline Nucleoplasty & 2 & $1^{\dagger}$ & 2 & 2 \\
\hline Ramus communicantes RF & 2 & 2 & 2 & 2 \\
\hline DRG pRF & 2 & 2 & 2 & 1 \\
\hline
\end{tabular}

*Procedure was repeated in 3 patients who had recurrence of pain at 3 months. ${ }^{\dagger}$ One patient had a delayed onset of pain relief. ${ }^{\ddagger} 2$ patients did not receive any pain relief and were subsequently lost to followup. 


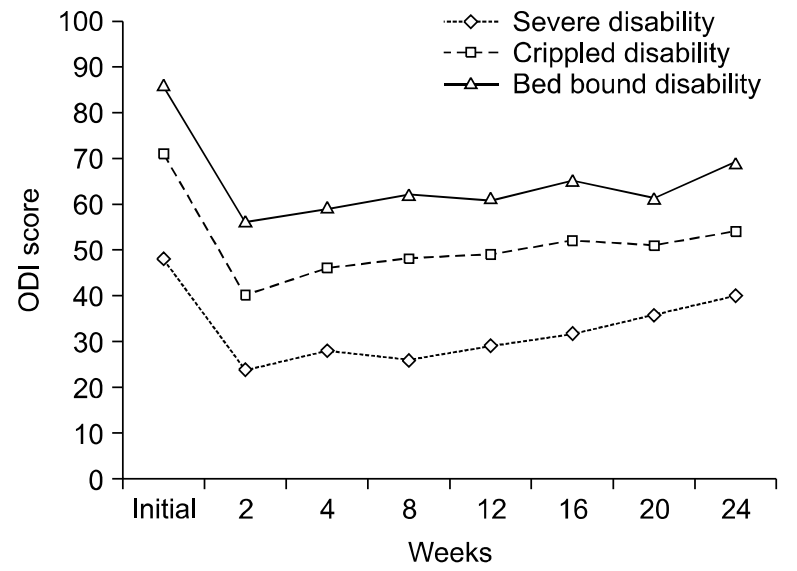

Fig. 1. Graphical presentation of the Oswestry disability scores over the follow-up period. The patients showed improvement in disability and shifted from a higher disability category to lower one.

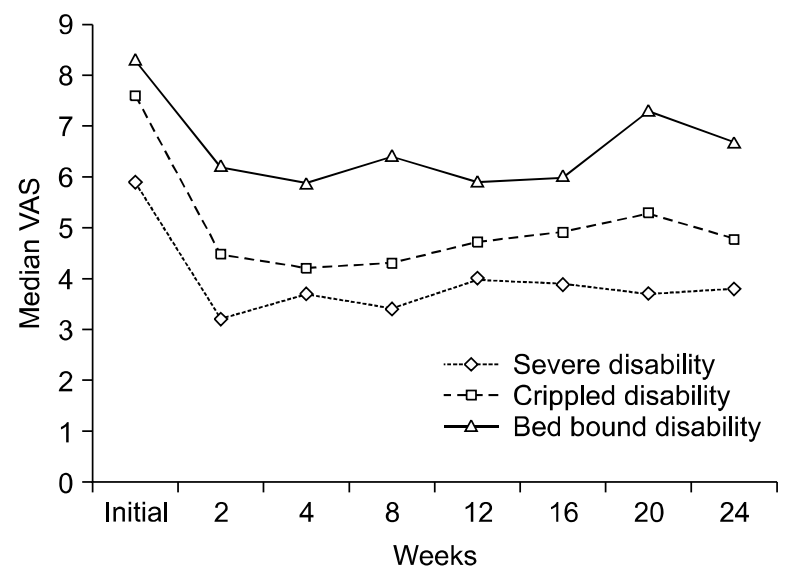

Fig. 2. Graphical presentation of the severity of pain on VAS during follow-up of the patients. Patients were sorted into groups based on the Oswestry disability index.

spine is excellent in identification of BS but may be inadequate in the cases of patients with milder hemisacralization. Six out of the 20 patients in the current study could not be identified on $\mathrm{X}$-ray; these patients were diagnosed only after bone scan and SPECT/CT were performed (Fig. 3). Therefore, inclusion of bone scan with SPECT/CT may prove to be an important tool in the armamentarium of pain physicians in evaluating patients with BS. Scoliosis on $\mathrm{X}$-ray is the most consistent feature that was identified in all 20 study patients.

The second most consistent finding in the present study was the origin of back pain from the facet joint. In most of the patients it was the L5-S1 facet on the ipsi-

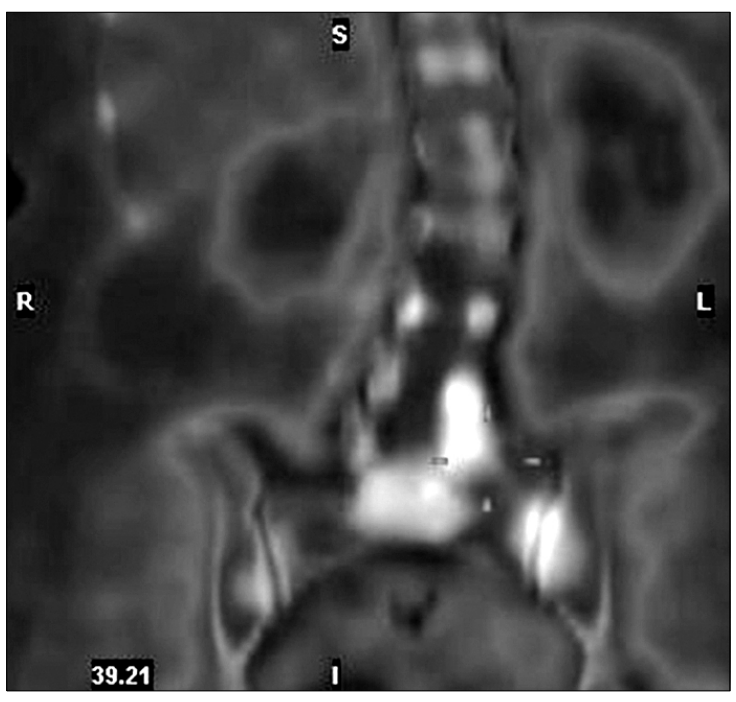

Fig. 3. SPECT/CT image of a patient of Bertolotti syndrome revealing Scoliosis. Increased tracer uptake at the left L4-5 and L5S1 facet joint and at left sacroiliac joint suggestive of inflammatory pathology at the respective sites.

lateral side. The contralateral facet may be strained owing to abnormal biomechanics due to scoliosis. Bone scan can reveal increased uptake at a particular facet joint, thereby pinpointing a particular facet joint when more than one facet is under consideration as the source of pain. We found SPECT/CT helpful in the evaluation of pain of facet joint origin (Fig. 3).

Sacroiliitis is also an important cause of low back pain in patients with $\mathrm{BS}$, and since this sacroiliitis probably originates due to abnormal pelvic tilt as a result of scoliosis, it is less likely to respond to steroid injection. Therefore, a RF denervation of the ipsilateral SI joint is a more prudent approach to management of sacroiliac joint pain in patients with BS. A bone scan with SPECT/CT can be of assistance in the identification of sacroiliitis (Fig. 3) [5].

Neoarticulation between the transverse process and the ilium is an additional pain-generating site in some patients. Steroid injection in the neoarticulation may provide temporary relief which may last up to two years [6]. In our observation, the neoarticular pain was difficult to treat. Some physicians have tried steroid and local anesthetic injection into the neoarticulations [6], excision of the neoarticulation, and posterolateral fusion of the transitional segment [7].

Disc degeneration is an important component in the pathophysiology of LBP in BS. The intervertebral disc most 
commonly involved is that of the L4-5 level, i.e., the level just above the level of hemisacralization. The possible explanation for this seems to be the restricted mobility at the level of hemisacralization leading to hypermobility at a level just superior to it. This hypermobility predisposes the intervertebral disc to degeneration.

According to Nakamura et al., sensory information from the lumbar intervertebral discs is transmitted to the rami communicantes via the sinuvertebral nerve. The rami communicantes then connect to the paravertebral sympathetic chain. This afferent information from the paravertebral chain is then transferred via the L1 and L2 communicating rami and the dorsal root ganglia of L1 and L2 to the spinal cord [8]. This information forms the basis for the emergence of rami communicantes block as a treatment modality for management of discogenic pain. RF lesioning of the grey ramus at vertebral levels adjacent to the painful disc or the white ramus at $\mathrm{L} 1$ and $\mathrm{L} 2$ vertebral level can be a good technique to provide palliation in $\mathrm{pa}^{-}$ tients with discogenic pain.

To date, there is no agreement as to the best method of treatment for BS patients. LBP in BS has been addressed by various methodologies but there is no consensus regarding definitive management. Consequently, the management of these patients remains uncertain. The absence of a consensus is of greater significance given the incidence of BS. Considering the economic and social impact of low back pain in young people, our findings highlight the importance of including BS in the differential diagnosis when examining young people with low back pain.

As far as the management of BS is concerned, one needs to develop a dynamic approach to pain management.
Multiple interventions may be required to achieve and maintain adequate pain relief. In some patients, pain relief may not be attained even after maximum possible efforts. Psychological counseling may be of great help and needs to be explored further.

\section{REFERENCES}

1. Aihara $T$, Takahashi $K$, Ogasawara A, Itadera E, Ono $Y$, Moriya $H$. Intervertebral disc degeneration associated with lumbosacral transitional vertebrae: a clinical and anatomical study. J Bone Joint Surg Br 2005; 87: 687-91.

2. Tae HS, Kim SD, Park JY, Kim SH, Lim DJ, Suh JK. Gray ramus communicans nerve block: a useful therapeutic adjuvant for painful osteoporotic vertebral compression fracture. J Korean Neurosurg Soc 2003; 34: 505-8.

3. Simopoulos TT, Malik AB, Sial KA, Elkersh M, Bajwa ZH. Radiofrequency lesioning of the $L 2$ ramus communicans in managing discogenic low back pain. Pain Physician 2005; 8: $61-5$.

4. Manchikanti L, Helm S, Singh V, Benyamin RM, Datta S, Hayek SM, et al. An algorithmic approach for clinical management of chronic spinal pain. Pain Physician 2009; 12: E225-64.

5. Henley C, Wollam K, Springs C. Sacroiliac pain: a physical therapy perspective. Post-Polio Health 2006; 22: 1-3.

6. Marks RC, Thulbourne T. Infiltration of anomalous lumbosacral articulations. Steroid and anesthetic injections in 10 back-pain patients. Acta Orthop Scand 1991; 62: 139-41.

7. Santavirta S, Tallroth K, Ylinen P, Suoranta H. Surgical treatment of Bertolotti's syndrome. Follow-up of 16 patients. Arch Orthop Trauma Surg 1993; 112: 82-7.

8. Nakamura SI, Takahashi K, Takahashi Y, Yamagata M, Moriya $\mathrm{H}$. The afferent pathways of discogenic low-back pain. Evaluation of L2 spinal nerve infiltration. J Bone Joint Surg Br 1996; 78: 606-12. 